\title{
A population viability analysis (PVA) for Cabot's Tragopan (Tragopan caboti) in Wuyanling, south-east China
}

\author{
YANYUN ZHANG and GUANGMEI ZHENG
}

\begin{abstract}
Summary
Understanding the status of fragmented populations and predicting their fate is an increasingly important part of bird conservation. Population viability analysis (PVA) can help in this process and is widely used for assessing the extinction risk faced by threatened species and for finding the key factors affecting population status and survival prospects. From 1982 to 2004, 14 scientists studied the population of the globally threatened Cabot's Tragopan Tragopan caboti in Wuyanling National Natural Reserve (WNNR), south-east China and collected life-history data on the population. Using VORTEX, we analysed the viability of the population in the reserve and this predicted that the population size will increase for the next 50 years and will then show a very slight decline for the next 50 years. The loss of heterozygosity is predicted to be $14 \%$, suggesting that the population may not be viable in the long term. Sensitivity analyses showed that nest loss is the most important factor affecting population size and the survival probability of the population, which is supported by field studies. Though the new evidence shows that Cabot's Tragopan can build nests in spruce forest successfully, broad-leaf forest is still necessary for them for foraging, especially at some times of the year. The simulation also shows that the probability of survival and the size of the population will decrease markedly if the extent of suitable habitat is reduced even relatively slowly (such as 0.1\% per year). Overall, we conclude that the PVA has provided very informative guidance to future management and research on Cabot's Tragopan at Wuyanling National Nature Reserve.
\end{abstract}

\section{Introduction}

An increasing number of wildlife populations are threatened by human activities and natural disasters. For example, $12 \%$ of the world's bird species are at risk of extinction, six bird species have become extinct over the last two decades and a further eight are probably so (IUCN 2004), mainly because of human pressures. Habitat loss and fragmentation is the principal cause of this high level of threat and is considered to be a major problem for birds specifically ( 1,045 globally threatened birds affected by this) and indeed for all threatened species (IUCN 2004).

Therefore, assessment of the potential impact that threats pose to the persistence of a species and its constituent populations is a major concern for conservation biologists (Soulé 1985, Brook and Kikkawa 1998, Hendrickson et al. 2003, Onal 2003). Population viability analysis (PVA) has been used for more than 25 years to explore the fate of populations under various scenarios (e.g. Shaffer 1981, Song 1996, Groom and Pascual 1998, William et al. 2002, Brook et al. 2002). It is an integrated process that considers almost all the factors that affect the process of species extinction, such as demographics, environmental conditions, genetics and catastrophes, as well as life history, dispersal and migration, and habitat use parameters (Gilpin and Soulé 1986, Ruggiero et al. 1994). This approach focuses on the likely fate of a population and which factors 
can determine or alter that fate. It is a type of risk assessment designed to project the likelihood of a population's persistence under particular conditions (Johnson and Braun 1999) and can thus also help to identify the most important factors faced by the endangered species (Boyce 1992). PVA is used as a decision-support tool, and it is important that predicted changes in the risks of extinction due to management are reliable, that the relative risks faced by different species are predicted accurately, and that the models can be used to help decide the most effective management strategy (McCarthy et al. 2003). Now PVA is widely used in conservation biology to predict extinction risk for threatened species and to compare alternative options for their management. Its predictions have been demonstrated to be relatively robust and unbiased, given adequate data (Brook et al. 2000). The IUCN Red List has such quantitative predictions as one of its five criteria for evaluating threatened status (IUCN 2001). The uncritical acceptance of PVA outputs has, however, been questioned, given that there is almost always uncertainty involved in predicting the probability of a species or population going extinct (Taylor 1995, Ludwig 1999, Coulson et al. 2001, Ellner et al. 2002). It is therefore very important that all the data for which there is uncertainty are considered, quantified, and reported if a PVA is performed (Winship and Trites 2006). Nonetheless, a retrospective study of 21 long-term studies concluded that predictions made using PVA were "surprisingly accurate" and that it is an appropriate tool for helping to manage populations of threatened species (Brook et al. 2000).

As in many parts of the world, wildlife populations in south-east China are increasingly fragmented and it is clear that the use of PVA will help assessment of their long-term survival prospects and thus inform their management. However, the availability of sufficient data to be informative and meaningful is currently very rare. Therefore, where such data do exist it is important to explore the value and limitations of this approach in future conservation planning for the species. One species for which there is a large body of appropriate data is Cabot's Tragopan (Tragopan caboti), a globally threatened pheasant endemic to China, where it is known as the Yellow-bellied Tragopan. It is considered Vulnerable (BirdLife International 2005), it is listed in the first category of nationally protected wildlife species in China and is included on CITES Appendix I (Zheng and Wang 1998, BirdLife International 2005).

The species has been studied in Wuyanling National Nature Reserve (NNR) for more than 23 year, in the longest-term study on any bird population in China. Given this research history, its conservation status and the fragmentation of populations, it is timely to assess the conservation prospects of this Cabot's Tragopan population. Therefore, in this paper, we: (I) review the information on Cabot's Tragopan in Wuyanling NNR, (2) predict its survival; and (3) determine the key factors affecting the intensively studied population. Finally, we comment on both the usefulness of this approach and how informative it is for conservation planning for the species.

\section{Methods}

\section{Review of population information}

Wuyanling National Nature Reserve (NNR) lies in southern Zhejiang Province, south-east China $\left(27^{\circ} 40^{\prime} 43^{\prime \prime} \mathrm{N}, 119^{\circ} 38^{\prime} 41^{\prime \prime} \mathrm{E}\right)$. Researchers from the College of Life Sciences at Beijing Normal University have studied this population for about 23 years (Table I).

Data have been gathered from Wuyanling NNR on age structure, general habitat and diet, population density, population size and sex ratio, dispersal and mortality, mating system, clutch size and nest loss, movements and home range, habitat use and nest-site selection and supplement. A captive breeding population has been established at Beijing Normal University for 20 years and this has allowed detailed information to be gathered on the development of the young, minimum and maximum breeding age of each sex and other aspects of reproductive biology. 
Table 1. List of relevant research literature on Cabot's Tragopan in Wuyanling NNR.

\begin{tabular}{lll}
\hline Author (in date order) & Year published & Subject \\
\hline Zheng et al. & 1985 & Breeding ecology \\
Zheng et al. & 1986 & Development of young \\
Zheng & 1987 & Habitat and population biology \\
Zhang and Zheng & 1988 & Feeding ecology \\
Zhang et al. & 1989 & Feeding activity \\
Zheng et al & $1989 b$ & Home range \\
Zhang and Zheng & 1989 & Nest selection and nest mortality \\
Zheng et al. & 1989 a & Courtship display \\
Zhang & 1987 & Population structure and dynamics \\
Zhang and Zheng & 1990 & Population biology \\
Qian & 1991 & Habitat selection \\
Young et al. & 1991 & Home range \\
Sun & 1990 & Home range, activity patterns, population characteristics \\
Sun and Zheng & 1992 & Home range and dispersal \\
Ding & 1993 & Mating system, supplementation, breeding ecology, \\
& & nest-site selection \\
Qian and Zheng & 1993 & Habitat selection \\
Gao & 1993 & Habitat characteristics, home range \\
Ding and Zheng & 1996 & Supplementation \\
Ding and Zheng & 1997 & Nest-site selection \\
Zheng and Wang & 1998 & Conservation assessment \\
Zhang and Zheng & 2001 & Assessment of reproductive condition \\
Zhang and Zheng & 2002 & Semen research \\
Deng and Zheng & 2004 & Habitat fragmentation \\
Deng et al. & 2005 & Artificial nest use \\
Zhang & 2005 & Review \\
\hline & & \\
\hline & &
\end{tabular}

Predicting the survival of the population

PVA is a type of risk assessment designed to project the likelihood of a population's persistence or, more directly, its extinction under particular conditions. Many models have been developed, such as GAPPS, SPGPC, RAMAS, FORPOP, ALEX, SIMPOP and VORTEX, of which VORTEX is the most widely used (Li et al. 1996, Brook and Kikkawa 1998, Armstrong and Ewen 2002). Here, VORTEX (Lacy 1993) was used to analyse the population viability by projecting population persistence over 100 years for the population of Cabot's Tragopan present in Wuyanling NNR. One thousand simulations were performed for each set of parameters.

\section{Sensitivity analysis}

Not all parameters that are included in the PVA model are likely to be equally important in influencing the population's long-term viability. Therefore, sensitivity analysis can help in identifying which parameters are key in reducing extinction risk, so the impact of a change of similar level in selected parameters was assessed using VORTEX. The sensitivity of predictions to the following parameters was analysed: loss of nests, yearling mortality, juvenile ( $1-2$ years) mortality, adult ( $>2$ years) mortality, fluctuation of the population from one year to the next, population carrying capacity and the occurrence of catastrophes. This was done using the following equation:

$$
S x=(\Delta x / x) /(\Delta P / P) \text { (Pulliam et al. 1992) }
$$

where $\Delta x / x$ is the change in population size resulting from a change of $\Delta P / P$ in the parameter $P$. In addition to analysing the sensitivity of predictions of the population size $(S x)$ to changes in 
the parameters selected, Pulliam's model was also used to analyse the sensitivity of predictions of extinct probability $(\mathrm{Se})$ in the equation

$$
S e=(\Delta e / e) /(\Delta P / P)
$$

where $\Delta e / e$ is the change in extinction probability resulting from a change of $\Delta P / P$ in the parameter $P$. Because a change of parameters does not affect the population size and the probability of survival in a linear fashion, there is no universal standard level at which $P / P$ should be set for a sensitivity analysis (McCarthy et al. 1995, Brook and Kikkawa 1998; Johnson and Braun 1999). Here the level of $\Delta P / P$ as $2 \%$ was selected to assess sensitivity.

Setting a level for the occurrence of catastrophic events was not straightforward as they are by nature rare, despite events such as fire, storm and serious drought being thought to have a large effect on the survival of Cabot's Tragopan (Zheng et al. 1985). Following discussion with the county government, this level was set at once in 100 years.

\section{Key factors}

While the sensitivity analysis reveals that parameters have a greater impact when altered by an arbitrarily chosen amount, the real impact produced by changes in parameters requires that the changes better reflect actual levels of variation. Therefore, field data from the studies referred to above were used to determine what these realistic levels of change should be for a subset of the parameters used above. It was decided that there were insufficient data available upon which to predict changes in mortality at individual ages and they were, therefore, excluded from this analysis. VORTEX was then used to predict population size and the survival probability of the population based on these more realistic levels of change in these parameters.

For a small population, inbreeding depression may play an important role in determining its future survival prospects. The impact of inbreeding was assessed by predicting the extinction probability and population size after 100 years if the population was not subject to inbreeding depression.

\section{Results}

\section{Cabot's Tragopan in Wuyanling NNR}

Population size

Population studies between 1983 and 1991 suggested that the average population size of Cabot's Tragopan in Wuyanling NNR was 85.0 (SD 10.2). The sex ratio of the population is close to 1 :1.

\section{Migration and dispersal}

Surrounded by farmlands and bamboo forests, Wuyanling is effectively a habitat island. Cabot's Tragopan has never been found in farmland or bamboo forests so it is suspected that the species cannot move through them. Altogether, 23 individuals have been tracked by radio-telemetry and no dispersal events were recorded.

\section{Reproduction}

Cabot's Tragopan has a monogamous mating system. Data from 30 nests show the following clutch sizes (mean 3.30, SD 0.2): 2 eggs, $7 \%$; 3 eggs, $48 \%$; 4 eggs, $33 \%$; 5 eggs, $4 \%$; and 6 eggs $7 \%$. Between 1983 and 1993 the overall nest loss was $48 \%$ (SD o.19), mainly as a result of predation by Eurasian Jay Garrulus glandarius, Yellow-throated Marten Charronia flavigula 
and Leopard Cat Felis bengalensis, or from disturbance by human activity. Because all eggs are destroyed in a predation event, this implies that in any year an average of $48 \%$ of females have failed nests.

Though the plumage of both sexes of year-old Cabot's Tragopan resembles that of the adults, those held in captivity have not bred until they were at least 2 years old. Indeed, male Cabot's Tragopan in captivity do not produce semen, and females do not lay well-formed eggs, until they are 2 years old. However, a few yearling females have laid one or two eggs during the breeding season. The maximum reproductive age of both sexes in captivity is 13 years old.

\section{Mortality}

Data available from radio-tracking a total of 23 birds indicated that mortality of adults and yearlings combined is $16 \%$.

\section{Carrying capacity}

The home range of Cabot's Tragopan is $0.015-0.3 \mathrm{~km}^{2}$ and there is $14 \mathrm{~km}^{2}$ of suitable habitat within the reserve. On the basis of suitable habitat, food availability and home range, we estimate the carrying capacity of Wuyanling NNR to be $300 \pm 50$ (SD).

\section{Predicting the survival of the study population}

The viability of the population

VORTEX predicted that there was a $7.3 \%$ probability that the Cabot's Tragopan population in Wuyanling NNR would be extinct in 100 years and that the mean loss of heterozygosity would be $14.0 \%$ (Figure 1 ). The mean inbreeding coefficient after 100 years is predicted to be $0.34 \%$. The prediction is that population size will almost double in to years and reach a peak of about

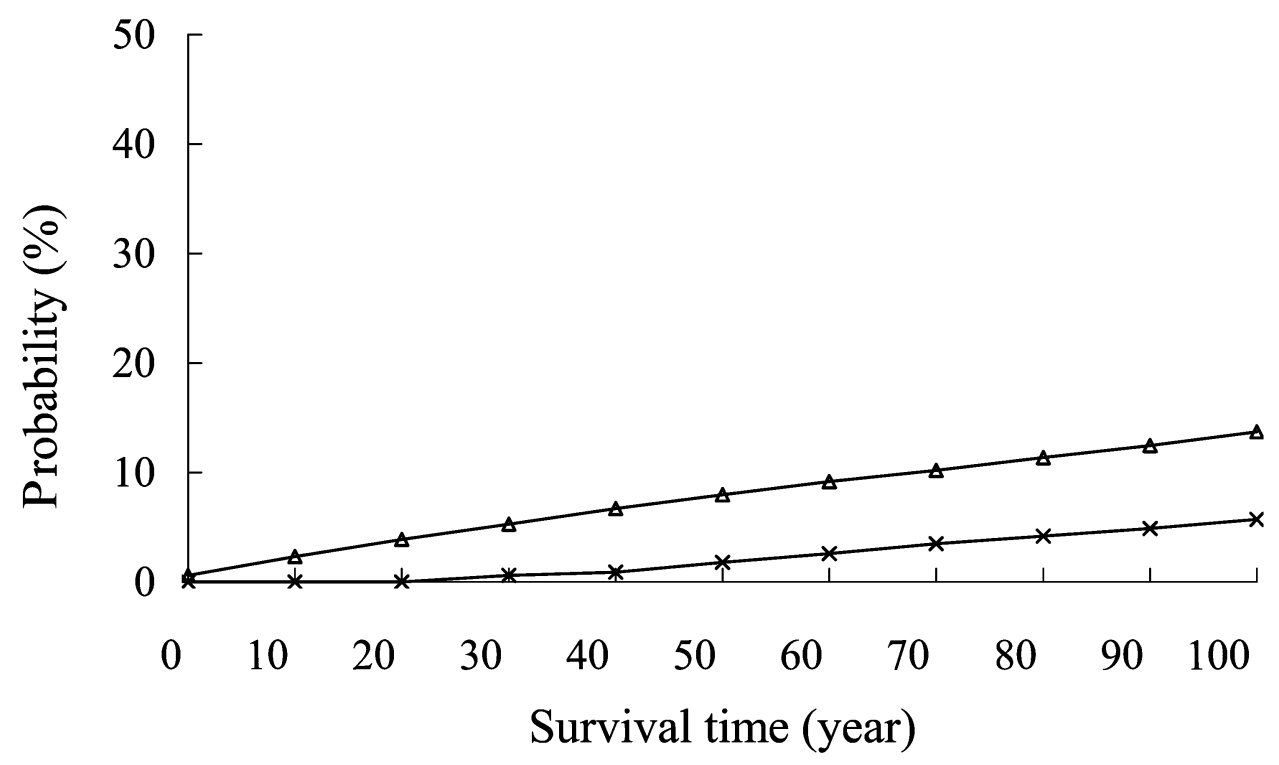

Figure 1. The probability of extinction and heterozygosity loss of the population of Cabot's Tragopan in Wuyanling NNR in the next 100 years. Line marked with $\triangle$ represent probability of extinction, and with $\times$ represent probability of heterzygosity loss. 


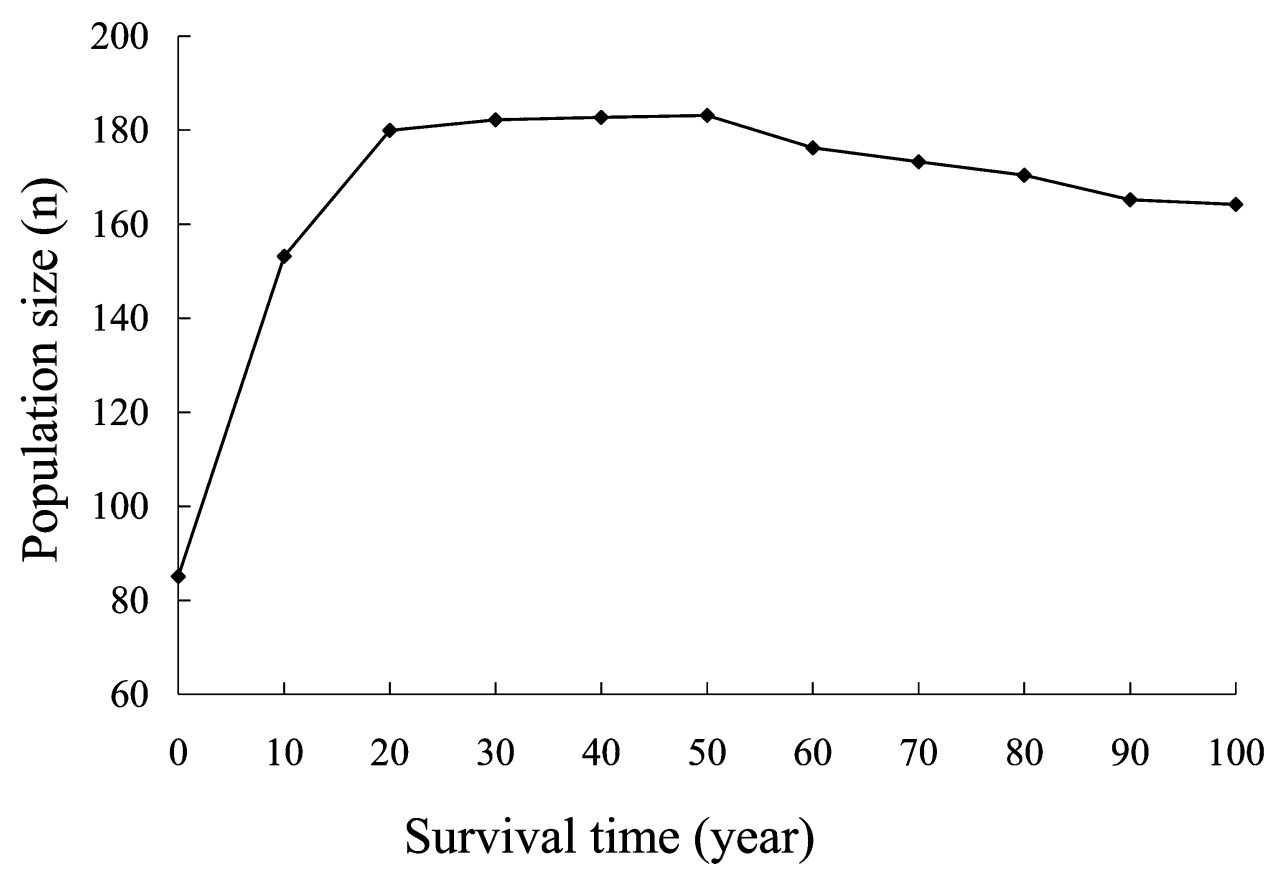

Figure 2. Predicted trend in population size of Cabot's Tragopan in Wuyanling NNR in the next 100 years.

180 birds in between 20 and 50 years before showing a slight decrease to around 165 birds in years $90-100$ (Figure 2). The long-term prediction is that the population has a $50 \%$ chance of extinction after 370 years.

\section{Determining the key factors}

\section{Sensitivity analysis}

The estimates of changes in the population size and the probability of extinction are sensitive to a $2 \%$ change in the selected parameters at three levels. They are most sensitive to nest loss and yearling mortality, followed by juvenile mortality, adult mortality and annual population fluctuation, with carrying capacity and catastrophes being least important (Table 2).

Table 2. Sensitivity analysis on parameters affecting the population size and survival probability of Cabot's Tragopan.

\begin{tabular}{lll}
\hline Parameters & $S x$ & $S e$ \\
\hline Nestling lost & 3.49 & 2.07 \\
Yearling mortality & 3.28 & 1.90 \\
Juvenile mortality & 1.07 & 1.69 \\
Adult mortality & 2.24 & 1.11 \\
Environmental variation & 2.13 & 1.01 \\
Carrying capacity & 0.15 & 0.21 \\
Catastrophic & 0.08 & 0.16 \\
\hline
\end{tabular}

Sx is the sensitivity of the population size on the basis of parameters changing; Se is the sensitivity of extinct on the basis of change in parameters. 
Table 3. The results of PVA of the Wuyanling NNR Cabot's Tragopan population, with the effects of change in carring capacity, environmental variability, nestling loss, and inbreeding depression.

\begin{tabular}{|c|c|c|c|}
\hline \multirow[t]{2}{*}{ Parameter } & \multicolumn{2}{|c|}{ Population size } & \multirow[t]{2}{*}{ Extinction probability } \\
\hline & Mean & $\mathrm{SD}$ & \\
\hline Current population $^{a}$ & $165 \cdot 3$ & 89.6 & $5.7 \%$ \\
\hline Carrying capacity reduced by $0.1 \%$ per year & 143.7 & 79.6 & $8.4 \%$ \\
\hline Carrying capacity reduced by $0.5 \%$ per year & 52.4 & $39 \cdot 4$ & $12.8 \%$ \\
\hline Environment variability increased by $50 \%$ & 82.6 & 92.5 & $31.5 \%$ \\
\hline Environment variability Doubled & $44 \cdot 7$ & $75 \cdot 5$ & $54.2 \%$ \\
\hline Environment variability decreased by $50 \%$ & 234.7 & 50.1 & $0.4 \%$ \\
\hline Nestling loss decreased by $13 \%$ & 208.0 & 71.7 & $1.7 \%$ \\
\hline Nestling loss decreased by $50 \%$ & $223 \cdot 3$ & 57.6 & $0.2 \%$ \\
\hline Without inbreeding depression & 199.1 & 74.2 & $1.2 \%$ \\
\hline
\end{tabular}

${ }^{a}$ From the actual data.

\section{Key factors}

Results were obtained on the predicted impact of variation in three parameters on the future survival prospects of the Wuyanling NNR Cabot's Tragopan.

\section{Carrying capacity}

If $0.1 \%$ of the total suitable habitat of the pheasant were to be lost every year (this may be lower as judged by currently observed rates of change), the extinction probability will increase to $8.4 \%$. As a worst case, if $0.5 \%$ of suitable habitat were lost each year, the extinction probability will rise to $12.8 \%$, and there will be only about 50 individuals surviving after 100 years (Table 3 ).

\section{Nest lost}

Field studies suggest that nest failure appears to be the most serious problem for the survival of the population. Human activities (e.g. logging) destroyed about $13 \%$ of nests directly (Ding 1993). If this impact can be reduced or even stopped (i.e. overall nest loss reduced by $13 \%$ ) the extinction probability would be reduced to $1.7 \%$, and the population would increase to more than 200 after 100 years. If nest loss were decreased by $50 \%$ (i.e. by also reducing loss from natural predators), this probability falls to $0.2 \%$ (Table 3 ).

\section{Population fluctuation}

If population size fluctuated by $50 \%$ or more from one year to the next, the probability that the species will go extinct is more than $30 \%$ (Table 3 ). If this annual variation could be reduced by half, however, the risk of extinction will be lower than in any other scenario explored here (Table 2). If none of the above scenarios were present, the population size may well double, but would have $5.7 \%$ probability of extinction over the next 100 years. If inbreeding depression were removed, this probability decreases to $1.2 \%$ (Table 2 ).

\section{Discussion}

PVA showed that the loss of heterozygosity of the Cabot's Tragopan population of Wuyanling NNR is $14 \%$. Therefore, it will not satisfy the criterion of Soule et al. (1986) for a viable 
population, which is one that should maintain $90 \%$ heterozygosity for 200 years. This means that this population is not stable in conservation terms.

Sensitivity analyses revealed that nest loss is the most important factor affecting the population size and extinction risk of Cabot's Tragopan in Wuyanling NNR. Field investigations have shown that Cabot's Tragopan prefers to build its nest between a large branch and the trunk of a tree and it is not difficult for natural predators to detect and destroy the nest (Zheng et al. 1985, Zheng 1987, Ding 1993, Zheng and Wang, 1998). Sometimes logging activity also destroyed some nests directly (Ding 1993) and both these factors have had a significant impact on nest loss. Thus, to conserve this population in the long term, we should pay more attention not only to natural predators, but also to the cessation of logging. Reducing nest loss significantly will make a substantial difference to the long-term viability of the population. For example, a decrease in nest loss of $50 \%$ is predicted to result in the population maintaining $90 \%$ heterozygosity for 200 years, which would fulfil the definition of a viable population.

The simulation result shows that the probability of survival and the size of the population will decrease markedly even if there is a small decrease in carrying capacity (e.g. suitable habitat was lost at the rate of $0.1 \%$ per year). This suggests that habitat loss and modification will also play a role in the future of Cabot's Tragopan at Wuyanling NNR. Our recent field investigation found that natural forest continues to be cleared or modified as a result of demands for timber and coal. Although trees have been planted after logging activity, the progressive replacement of natural evergreen broadleaved forests with commercial coniferous forest is now a major problem for this species. Previously, it has been shown that the distribution of Cabot's Tragopan is closely associated with the tree Daphniphyllum macropodum, which is often used for roosting, and its leaves and fruit are a favoured food (Zheng, 1987, Zhang and Zheng 1988). Though recent evidence has shown that Cabot's Tragopan has begun to nest in spruce forests (Ding and Zheng 1997, Deng et al. 2005), this change may indicate a serious problem. The reason is that the berries, leaves and nuts of some species of broadleaved tree (e.g. Daphniphyllum macropodum, Fagus spp., Castanopsis sclerophylla) are the species' primary food, especially when food is in short supply in early spring and winter (Zheng et al.. 1985, Zheng 1987, Zhang and Zheng 1988, Qian and Zheng 1993). Without these broadleaved trees, Cabot's Tragopan cannot survive through the winter and so the maintenance of these forests is vital, together with a reduction in any disturbance during the nesting period.

In PVA, adequate data are absolutely necessarily because limited or poor quality data cannot imitate the "process" of population change (Ruggiero et al. 1994, Brook and Kikkawa 1998). In our case, identifying nest loss as a highly important factor, allows us to see how reducing nest loss significantly can lead to a reduction in extinction risk. Also, if we knew more about, for example, mortality of young, yearlings and adults, then we could increase the predictive ability of our analysis.

Because it is fairly difficult to collect high-quality data for many characteristics of a threatened population, especially parameters such as carrying capacity, catastrophe frequency and lifehistory data, it has been claimed that PVA has little useful predictive value and should be dispensed with in favour of alternative methods (e.g. Harcourt 1995; Coulson et al., 2001). Cabot's Tragopan in Wuyanling NNR is probably one of the most studied pheasant populations in Asia but even so there are many gaps in our knowledge. Bearing this in mind, has PVA been a useful analysis for this population? The answer has to be yes, because it has allowed us to predict future population trends and identify an important factor that is affecting extinction risk. This information can guide management decisions and future research. For example, PVA suggested that nest loss plays a very important role in influencing population viability, and this implies that we should pay more attention to solving this problem in future. Even if we do not have all the data that we would like in order to perform a viability analysis, the process is very useful as part of an adaptive management approach (see e.g. Salafsky et al. 2001) in the area, rather than providing a definitive assessment of the precise extinction risk of the population. Even with data 
of unknown accuracy, or with data from related species, it is possible to evaluate, to a degree, extinction risk and inform management strategies (Gaillard et al. 200o).

\section{Acknowledgements}

We thank the doctoral and masters students and the research assistants of the Pheasants Research Group of the College of Life Sciences, Beijing Normal University, for collecting longterm data of the Cabot's Tragopan population of Wuyanling area. P. McGowan, B. Brook, Li X. H., Ma Z. J. and R. Sumner provided many constructive suggestions, and H. R. Pulliam provided related papers. This work was supported by the National Natural Sciences Foundation of China (Nos. 30330050, 30670289).

\section{References}

Armstrong, D. and Ewen, J. (2002) Dynamics and viability of a New Zealand Robin population reintroduced to regenerating fragmented habitat. Conserv. Biol. 16: 1074-1085.

BirdLife International (2005) 2005 IUCN Red List Category. Available online at http:// www.birdlife.org/datazone/species/ index.html?action $=$ SpcHTMDetails.asp\&sid $=241 \& \mathrm{~m}=\mathrm{O} \#$

Boyce, S. (1992) Population viability analysis. Annu. Rev. Ecol. Syst. 23: 481-506.

Brook, B. and Kikkawa, J. (1998) Examining threats faced by island birds: a population viability analysis on the Capricorn silvereye using long-term data. J. Appl. Ecol. 35: 491-503.

Brook, B., Grady, J., Chapman, A., Burgman, M., Akcakaya, H. and Frankham, R. (200o) Predictive accuracy of population viability analysis in conservation biology. Nature 404: 385-387.

Brook, B., Burgman, M., Akcakaya, H., O'Grady, J. and Frankham, R. (2002) Critiques of PVA ask the wrong questions: throwing the heuristic baby out with the numerical bath water. Conserv. Biol. 16: 262-263.

Coulson, T., Mace, G. M., Hudson, E. and Possingham, H. (2001) The use and abuse of population viability analysis. Trends Ecol. Evol. 16: 219-221.

Deng, W. H. and Zheng, G. M. (2004) Landscape and habitat factors affecting Cabot's Tragopan Tragopan caboti occurrence in habitat fragments. Biol. Conserv. 117: 25-32.

Deng, W. H., Zheng, G. M., Garson, P. and McGowan, P. (2005) Providing artificial nest platforms for Cabot's Tragopan Tragopan caboti (Aves: Galliformes): a useful conservation tool? Oryx 39: 118-123.

Ding, C. Q. (1993) The mating system and the breeding ecology studies of Cabot's Tragopan. PhD thesis, Beijing Normal University. (In Chinese with English abstract.)

Ding, C. Q. and Zheng, G. M. (1996) A primary study on the reintroduction of Cabot's Tragopan. Acta Zool. Sin. 42 (Suppl.): 69-73. (In Chinese with English abstract.)

Ding, C. Q. and Zheng, G. M. (1997) A studying on the nest selection of Cabot's Tragopan. Acta Zool. Sin. 43: 27-33. (In Chinese with English abstract.)

Ellner, S. P., Fieberg, J., Ludwig, D. and Wilcox, C. (2002) Precision of population viability analysis. Conserv. Biol. 16: 258-261.

Gaillard, J. M., Festa-Bianchet, M., Yoccoz, N. G., Loison, A. and Toigo, C. (2000) Temporal variation in fitness components and population dynamics of large herbivores. Annu. Rev. Ecol. Syst. 16: 367-393.

Gao, Q. (1993) The habitat character and selection in autumn of Cabot's Tragopan. MSc thesis, Beijing Normal University. (In Chinese with English abstract.) 
Gilpin, M. E. and Soulé, M. E. (1986) Minimum viable population: processes of extinction. Pp. 19-34 in M. E. Soulé, ed. Conservation biology: the science of scarcity and diversity. Sunderland, MA: Sinauer.

Groom, M. and Pascual, M. (1998) The analysis of population persistence: an outlook on the practice of population viability analysis. Pp. $4^{-27}$ in P. L. Fieldler and P. M. Karieva, eds. Conservation biology for the coming decade. New York: Chapman and Hall.

Harcourt, A. H. (1995) Population viability estimates: theory and practice for a wild gorilla population. Conserv. Biol. 9: 134-142.

Hendrickson, S. L., Bleiweiss, R., Matheus, J. C., Matheus, L. S., Jacome, N. L. and Pavez, E. (2003) Low genetic variability in the geographically widespread Andean Condor. The Condor 105: 1-12.

IUCN (2001) IUCN Red List Categories. Gland, Switzerland, Cambridge, U.K.: IUCN.

IUCN (2004) 2004 IUCN Red List of Threatened Species. Gland, Switzerland, Cambridge, U.K.: IUCN.

Johnson, K. H. and Braun, C. E. (1999) Viability and conservation of an exploited Sage Grouse population. Conserv. Biol. 13: 77-84.

Lacy, R. C. (1993) Vortex: a computer simulation model for population viability analysis. Wildl. Res. 20: 45-65.

Li, X. H., Li, D. M., Lu, B. Z. and Zai, T. Q. (1996) A population viability analysis of Niponia nipon. Biodiversity 4: 69-77 (In Chinese with English abstract.)

Ludwig, D. (1999) Is it meaningful to estimate a probability of extinction? Ecology 80: 298-310.

McCarthy, M. A., Burgman, M. A. and Ferson, S. (1995) Sensitivity analysis for models of population viability. Biol. Conserv. 73: 93-100.

McCarthy, M. A., Andelman, S. J. and Possingham, H. P. (2003) Reliability of relative predictions in population viability analysis. Conserv. Biol. 17: 982-989.

Onal, H. (2003) Preservation of species and genetic diversity. Am. J. Agric. Econ. 85: 437-447.
Pulliam, H. R., Dunning, J. B. and Liu, J. (1992) Population dynamics in a complex landscape: a case study. Ecol. Application 2: 165-177.

Qian, F. W. (1991) The habitat selection of the Cabot's Tragopan. MSc thesis, Beijing Normal University. (In Chinese with English abstract.)

Qian, F. W. and Zheng, G. M. (1993) A studying on the habitat selection of Cabot's Tragopan. J. Beijing Normal Univ. (Nat. Sci.) 29: 256-264 (In Chinese with English abstract.)

Ruggiero, L., Hayward, G. and Squires, J. (1994) Viability analysis in biological evaluations: concepts of population viability analysis, biological population, and ecological scale. Conserv. Biol. 8: 364-372.

Salafsky, N., Margoulis, R. and Redford, K. (2001) Adaptive management: a tool for conservation practitioners. Washington, DC: Biodiversity Support Program.

Shaffer, M. (1981) Minimum population sizes for species conservation. Bioscience 31: 131-134.

Song, Y. L. (1996) Population viability analysis for two isolated populations of Hainan Eld's deer. Conserv. Biol. 10: 1467-1472.

Soulé, M. (1985) What is conservation biology? Bioscience 35: 727-734.

Soulé, M., Gilpin, M., Conway, W. and Foose, T. (1986) The millennium ark: how long a voyage, how many staterooms, how many passengers? Zoo Biol. 5: 101-113.

Sun, Y. H. (1990) A study on activity of Cabot's Tragopan using radio tracking method. MSc thesis, Beijing Normal University. (In Chinese with English abstract.)

Sun, Y. H. and Zheng, G. M. (1992) A radiotracking study of home range of Cabot's Tragopan. Chinese Acta Zool. Sinica 38: 385-389. (In Chinese with English abstract.)

Taylor, B. L. (1995) The reliability of using population viability analysis for risk classification of species. Conserv. Biol. 9: 551-558.

William, E. M., Philip, L. B., Brain, R. H., Leonie, C. M. and Johm, R. S. (2002) Population viability analysis in endangered species recovery plans: past use and future 
improvements. Ecol. Application 12: 708-712.

Winship, A. J. and Trites, A. W. (2006) Risk of extirpation of Steller's sea lions in the Gulf of Alaska and Aleutian Islands: a population viability analysis based on alternative hypotheses for why sea lions declined in western Alaska. Marine Mamm. Sci. 22: 124-155.

Young, L., Zheng, G. M. and Zhang, Z. W. (1991) Winter movements and habitat use by Cabot's Tragopan in southeastern China. Ibis 133: 121-126.

Zhang, J. P. (1987) A study on the population structure and dynamic of Cabot's Tragopan. MSc thesis, Beijing Normal University. (In Chinese with English abstract.)

Zhang, Z. and Zheng, G. M. (1988) Feeding ecology of Cabot's Tragopan. J. Beijing Normal Univ. (Nat. Sci.). 2 (Suppl.): 685-689. (In Chinese with English abstract.)

Zhang, J. P. and Zheng, G. M. (1990) Numbers and population structure of Cabot's Tragopan. Chinese Zool. Res. 11: 291-297. (In Chinese with English abstract.)

Zhang, Y. Y. (2005) The review of Cabot's Tragopan. Chinese J. Zool. 40: 104-107. (In Chinese with English abstract.)

Zhang, Y. Y. and Zheng, G. M. (2001) Study of the changing of the sex steroid extract from the droppings of Cabot's Tragopan (Tragopan caboti) in captivity. J. Beijing Normal Univ. (Nat. Sci.) 37: 685-689. (In Chinese with English abstract.)

Zhang, Y. Y. and Zheng, G. M. (2002) A study on the artificial insemination of Cabot's Tragopan (Tragopan caboti). J. Beijing Normal Univ. (Nat. Sci.) 38:
118-122. (In Chinese with English abstract.)

Zhang, Z. W. and Zheng, G. M. (1989) Nest site selection and nest mortality of Cabot's Tragopan in Wuyanling Natural Reserve, China. Pp. 48-53 in World Pheasant Association (WPA), ed. Pheasants in Asia. (In Chinese with English abstract.)

Zhang, Z. W., Yin, R. L. and Zheng, G. M. (1989) Feeding activity of the Cabot's Tragopan during the breeding season in captivity. Chinese Zool. Res. 10: 333-339. (In Chinese with English abstract.)

Zheng, G. M. (1987) Cabot's Tragopan. Chinese J. Zool. 22(5): 40-43. (In Chinese with English abstract.)

Zheng, G. M. and Wang, Q. S., eds. (1998) Pp. 157-159 in China red data book of endangered animals: Aves. Beijing: Science Press. (In Chinese with English abstract.)

Zheng, G. M., Zhao, X. R. and Song, J. (1985) On the breeding ecology of Tragopan caboti. Acta Ecol. Sinica 5: 379-385.

Zheng, G. M., Yin, R. L. and Zhang, Z. W. (1986) The reproduction and development of fledging of Cabot's Tragopan in captivity. Wildlife 6: 39-43. (In Chinese with English abstract.)

Zheng, G. M., Yin, R. L., Zhang, Z. W., Liu, Z. X. and Zhou, H. Q. (1989a) Behavior of courtship display of Cabot's Tragopan. Acta Zool. Sinica 35: 328-332. (In Chinese with English abstract.)

Zheng, G. M., Zhang, Z. W. and Young, L. (1989b) The use of radio-telemetry to study Cabot's Tragopan in Wuyanling Natural Reserve, China. Pp. 48-53 in World Pheasant Association (WPA), ed. Pheasants in Asia. (In Chinese with English abstract.)

\section{YANYUN ZHANG, GUANGMEI ZHENG*}

Ministry of Education Key Laboratory for Biodiversity and Ecological Engineering, College of Life Sciences, Beijing Normal University, Beijing100875, P. R. China.

*Author for correspondence; e-mail: zhenggm@bnu.edu.cn 